\title{
Estimation of lower airway resistance in expiratory flow limitation during mechanical ventilation
}

\author{
P. Barbini, G. Cevenini \& M. R. Massai \\ Department of Surgery and Bioengineering, Siena University, Italy
}

\begin{abstract}
Determining the level of expiratory flow limitation (EFL) in critical mechanically ventilated patients is of clinical relevance for choosing appropriate respiratory treatment and/or pharmacological therapy. In many respiratory diseases, EFL arises from pathological changes in the mechanical properties of the bronchial tree. These properties cannot be evaluated using common lumped-parameter models or conventional estimation approaches based on measured mouth pressure and flow data, because during EFL expiratory flow no longer depends on the difference between alveolar and mouth pressure (Starling resistor effect). Starting from simulation data obtained by a dynamic non linear lumped-parameter model recently proposed for interpreting the main effects of EFL, we developed a two-step procedure which analyses inspiration and expiration separately in mechanically-ventilated flow-limited patients, thus enabling total resistance of deeper airway generations influencing the onset of EFL to be estimated. The negative expiratory pressure method was used to detect the presence of flow limitation during expiration. The model-to-model approach showed that lung and chest wall elastances and resistance of the lower bronchial tree could be estimated with reasonable accuracy from mouth flow and mouth and pleural pressure data. The estimated values are consistent with the mechanical properties of the lower generations of the bronchial tree described by the forward model. On the basis of these estimated parameters, the end-expiratory occlusion technique also allows the end-expiratory lung volume to be determined.
\end{abstract}

Keywords: respiratory system, breathing mechanics model, expiratory flow limitation, mechanical ventilation, parameter identification, resistance. 


\section{Introduction}

Expiratory flow limitation (EFL) is a phenomenon with pathophysiological implications occurring in many respiratory diseases [1-2]. Patients with chronic obstructive pulmonary disease under mechanical ventilation for acute respiratory failure are critical cases requiring particular attention. In these patients, EFL must be detected and quantified in order to determine the best ventilatory and/or pharmacological approach.

The two basic mechanisms involved in the flow-limiting process are the wave-speed mechanism and the viscous mechanism [3]. The first mechanism is generally predominant at medium and high lung volumes when flow velocity approaches wave speed, whereas the second is predominant at low lung volumes [4]. During EFL, expiratory flow is independent of expiratory effort, i.e. of the difference between alveolar pressure and mouth pressure (driving pressure) [1]. To understand this phenomenon, it is useful to consider the pressures inside and outside the thoracic airways. In flow-limited patients pleural pressure (pressure outside the thoracic airways) can reach high values. Because of the pressure drop along the airways (from alveoli to mouth), during expiration high pleural pressures compress the airways downstream of the equal pressure point (EPP, the point where pleural pressure equals lateral pressure inside the bronchial tree). This phenomenon is known as expiratory dynamic compression of the airways (fig. 1). Under these conditions, flow is determined by the difference between alveolar pressure and pleural pressure rather than by driving pressure (Starling resistor effect).

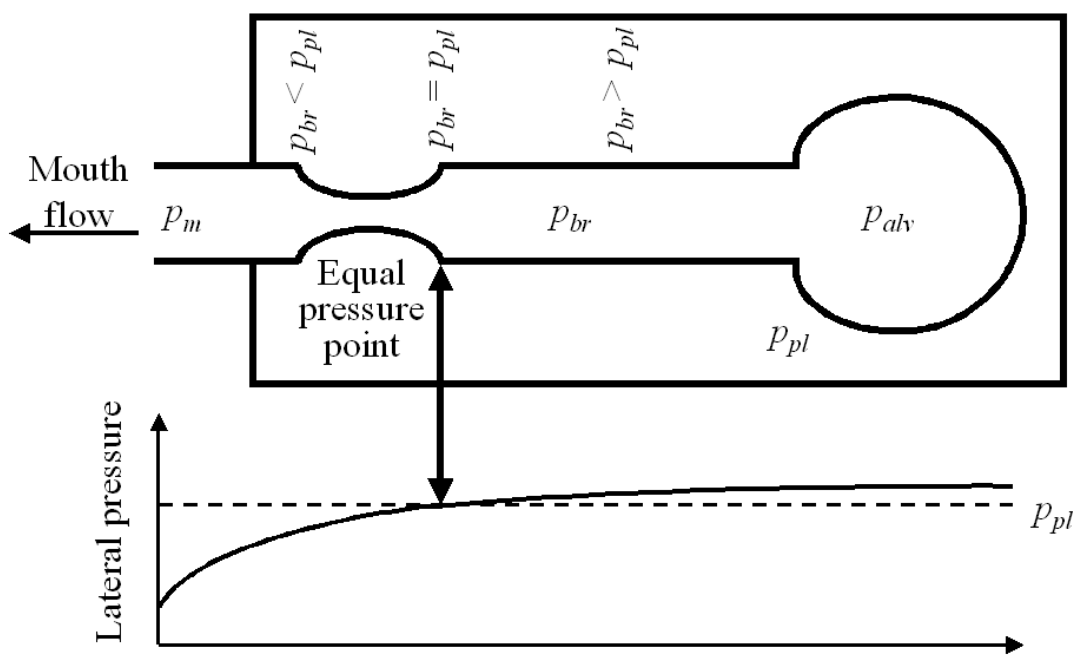

Figure 1: Schematic representation of EFL due to airways compression. $p_{m}$, $p_{p l}$, and $p_{a l v}$ indicate mouth, pleural and alveolar pressure, respectively, while $p_{b r}$ is the lateral pressure inside the bronchial tree. 
EFL is mainly caused by pathological changes in the mechanical properties of the lower airways [5]. It is difficult to assess these properties by standard techniques based on common lumped parameter models used during mechanical ventilation, because they cannot be identified from mouth pressure and flow data alone, because the Starling resistor effect renders expiratory flow independent of driving pressure and therefore of mouth pressure.

In this study we propose an approach for estimating the expiratory resistance of airway generations upstream of the EPP in mechanically-ventilated flowlimited patients on the basis of accessible pressure and flow data. The performance of the proposed method is evaluated by a model-to-model approach. An essential nonlinear lumped representation of breathing mechanics, recently employed to interpret EFL in mechanical ventilation [5], is used as forward model. From mouth pressure and flow data, we first obtained estimates of total lung resistance and elastance during inspiration, when flow limitation is absent. To do this we also determined the intrinsic positive end-expiratory pressure by the well-known end-expiratory occlusion technique. Mouth flow and intrapleural pressure data were then used to estimate resistance upstream of EPP during EFL. This final step also required knowledge of changes in alveolar pressure, which were determined assuming that differences between inspiratory and expiratory total pulmonary elastance were negligible [6-7].

\section{Methods}

\subsection{Forward model of breathing mechanics in mechanical ventilation}

Figure 2 shows the electrical analogue of the forward model representing EFL in mechanically-ventilated flow-limited patients [5]. $\dot{v}(t)$ is mouth flow and $p_{m}(t)$, $p_{p l}(t), p_{a l v}(t)$ and $p_{t m}(t)$ are mouth, pleural, alveolar and transmural pressure, respectively. In this model, the viscoelastic properties of the tracheobronchial airways are described by nonlinear relationships, whereas the behaviour of the alveolar space and chest wall are assumed to be linear.

The tracheobronchial airways are divided into three segments:

1) upper airways, characterized by a flow-dependent Rohrer resistor

$$
R_{u}=K_{1}+K_{2}|\dot{v}|
$$

where the constant $K_{1}$ represents resistive properties for laminar flow and constant $K_{2}$ represents an additional flow-dependent resistive term, which becomes significant at higher flow rates when turbulence may occur;

2) intermediate collapsible airways, described as a cylinder of constant length, having a volume $\left(v_{c}\right)$ varies with transmural pressure, according to the elastic characteristic

$$
\frac{v_{c}}{v_{c \max }}=\frac{1}{1+\exp \left[-a\left(p_{t m}-b\right)\right]}
$$


where $v_{c \text { max }}$ is maximum airway volume, and $a$ and $b$ are constants. The intermediate airway resistance is described by Poiseuille's law

$$
R_{i}=K_{3}\left(\frac{v_{c \max }}{v_{c}}\right)^{2}
$$

and its elastance $E_{a w}$ can be obtained differentiating $p_{t m}$ with respect to $v_{c}$.

3) lower airways, represented by a resistance $\left(R_{l}\right)$ depending on lung volume $v$

$$
R_{l}=\frac{K_{4}}{v}
$$

where $K_{3}$ and $K_{4}$ are constants and $v$ depends on tidal volume and end-expiratory lung volume.

The alveolar space and chest wall are assumed to be purely elastic and are represented by constant elastances $E_{p}$ and $E_{c w}$, respectively.

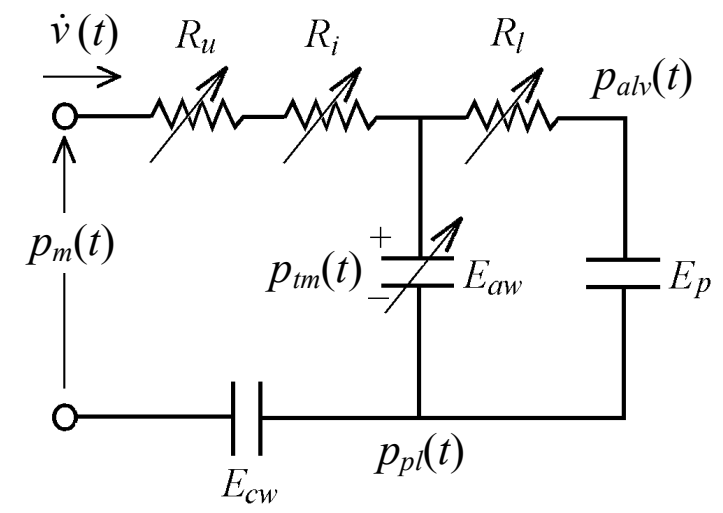

Figure 2: Electrical analogue of the non-linear model of breathing mechanics.

The mechanical ventilator was modelled as an inspiratory positive-pressure generator with high internal resistance. A short post-inspiratory pause with zero mouth flow was also reproduced, followed by passive expiration. No external positive end-expiratory pressure was simulated.

EFL was assessed by analysing changes induced in the flow-volume loop by a negative expiratory pressure (NEP) applied at the airway opening. The NEP method was simulated applying a sub-atmospheric pressure at the airway opening, beginning $40 \mathrm{~ms}$ after peak expiratory flow, and comparing the ensuing expiratory flow-volume curve with that of the previous control expiration [2]. Simulations were performed with Matlab-Simulink software. 


\subsection{Model identification procedure}

Estimation of key viscoelastic parameters characterizing breathing mechanics in artificially-ventilated flow-limited patients is difficult, especially in patients with severe airway obstruction [8]. In these cases parameter estimates are usually based the inspiratory phase only [9].

In less critical conditions, the well-known inverse model of breathing mechanics shown in fig. 3 is used in clinical practice to estimate total respiratory resistance $R$ and elastance $E$ during a whole breathing cycle on the basis of mouth pressure and flow data [7]. However, in this case too, it has been proved that it is preferable to consider the inspiratory and expiratory phases separately, in order to improve the precision and accuracy of the estimates [6-7]. In particular, estimated total inspiratory resistance, $R_{\text {insp }}$, has been found significantly different from estimated total expiratory resistance, $R_{\text {exp }}$, whereas estimated total inspiratory and expiratory elastances do not show any statistically significant differences.

On the basis of the above considerations, we developed an estimation approach for identifying important features of the system even in expiratory phase of mechanically-ventilated flow-limited patients.

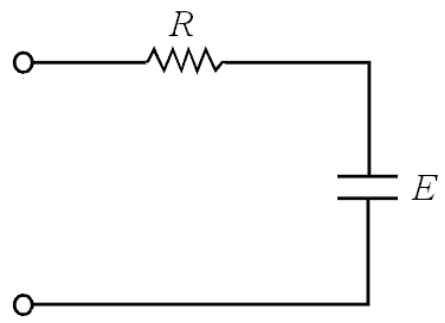

Figure 3: Simple one-compartment model of breathing mechanics.

\subsubsection{Inspiratory parameter estimation}

Using the inverse model of fig. 3, total respiratory resistance $\left(R_{\text {insp }}\right)$ and elastance $\left(E_{\text {insp }}\right)$ were estimated during inspiration from inspiratory mouth pressure $p_{m}(t)$ and flow $\dot{v}(t)$ data. On the basis of previous findings [6-7] $E_{\text {insp }}$ was assumed equal to total respiratory elastance $E$ during the whole breath.

Use of this simple RE inverse model is based on the assumption that system behaviour during inspiration can be described by the following relationship

$$
p_{m}(t)=R_{\text {insp }} \dot{v}(t)+E\left[v_{0}+\Delta v(t)\right]
$$

where $v_{0}$ is the difference between the end-expiratory lung volume (EELV) and the functional residual capacity (FRC) and $\Delta v(t)$ is the change in lung volume during a tidal breath, obtained by integrating mouth flow.

The product $E v_{0}$ is the value of $p_{m}$ when $\dot{v}$ and $\Delta v$ are zero. In clinical practice, it is known as the intrinsic positive end-expiratory pressure (PEEPi). 
Equation 5 can be rewritten

$$
p_{m}(t)-\mathrm{PEEPi}=R_{\text {insp }} \dot{v}(t)+E \Delta v(t)
$$

In mechanically ventilated patients, PEEPi can be measured by the endexpiratory occlusion technique [10]. The above relationship, describing the inspiratory behaviour of the system, is therefore a linear regression model, the unknown parameters ( $R_{\text {insp }}$ and $E$ ) of which can be estimated from mouth pressure, PEEPi, mouth flow and its integral. Of course, if we know $E$ and FRC we can estimate EELV as well. EELV is a very important parameter to know for the respiratory treatment of flow-limited patients.

Finally, with reference to the forward model of fig. 2, note that the estimated value of $E$ is a reasonable approximation of the sum of pulmonary $\left(E_{p}\right)$ and chest wall elastance $\left(E_{c w}\right)$, i.e.

$$
E=E_{c w}+E_{p}
$$

due to very high values of $E_{a w}$ over the whole breath. If changes in pleural pressure $\left(\Delta p_{p l}\right)$ are measured during the tidal breath, it is possible to estimate $E_{c w}$ by the following equation

$$
\Delta p_{p l}(t)=E_{c w} \Delta v(t)
$$

and this provides distinct estimates for $E_{p}$ and $E_{c w}$.

\subsubsection{EFL parameter estimation}

In flow-limited patients, transmural pressure at EPP is zero during expiration. In other words, at this point the pressure inside the airways is equal to pleural pressure. Under these conditions expiratory flow in deeper airways (from alveoli to EPP) is determined by the difference between alveolar $\left(p_{a l v}\right)$ and pleural pressure $\left(p_{p l}\right)$. Furthermore, because of the above-described Starling resistor effect, it equals expiratory mouth flow $\dot{v}(t)$. Modelling all airways from EPP to the alveoli with a single constant resistance $\mathrm{Rd}$, we have the following limited expiratory flow

$$
-\dot{v}(t)=\frac{p_{a l v}(t)-p_{p l}(t)}{R_{d}}
$$

where the minus sign is because we assume positive airflow during inspiration. Since only pleural pressure time-changes (i.e., $\left.\Delta p_{p l}(t)=p_{p l}(\mathrm{t})-p_{p l}(0)\right)$ can be measured in the clinic, and this data allows us to obtain distinct estimates for $E_{c w}$ and $E_{p}$, it is convenient to rearrange eqn. 9 as follows

$$
\left[p_{p l}(t+T)-p_{p l}(t)\right]-\left[p_{a l v}(t+T)-p_{a l v}(t)\right]=R_{d}[\dot{v}(t+T)-\dot{v}(t)]
$$

where $t$ and $T$ are discrete time and sampling interval, respectively. 
During a tidal expiration, we can assume

$$
p_{p l}(t+T)-p_{p l}(t)=E_{c w}[\Delta v(t+T)-\Delta v(t)]
$$

and

$$
p_{a l v}(t+T)-p_{a l v}(t)=\left(E_{c w}+E_{p}\right)[\Delta v(t+T)-\Delta v(t)]
$$

where

$$
\Delta v(t+T)-\Delta v(t)=T \dot{v}(t)
$$

From eqns. 11-13, eqn. 10 can be rewritten

$$
\dot{v}(t+T)=A \dot{v}(t)
$$

where

$$
A=1-\frac{E_{p} T}{R_{d}}
$$

The above relationship can be used to estimate the unknown parameter $A$ and, consequently $R_{d}$. Note that eqn. 14 is valid only in expiratory tracts with EFL, which can be detected by the NEP technique [2].

\section{Results and conclusion}

Table 1 shows the set of parameter values of the forward model chosen for the simulation of a mechanically ventilated flow-limited patient. This set of values gives rise to EFL over most of the expiration [5].

We simulated the end-expiratory occlusion technique and calculated PEEPi as the difference between the mouth pressure at the end of occlusion and the external positive end-expiratory pressure (zero in the present case), namely $7.7 \mathrm{cmH}_{2} \mathrm{O}$, giving an end-expiratory lung volume of 3.39 1, i.e. $13 \%$ greater than the set value of FRC.

On the basis of estimated PEEPi and inspiratory mouth pressure and flow data, we estimated the values of $R_{\text {insp }}$ and $E$ and the corresponding $95 \%$ confidence interval (table 2).

Comparing the estimated value with the parameters of the forward model we observe that the estimate of $E$ properly approximates the sum $E_{c w}+E_{p}$. In particular, this sum falls within the $95 \%$ confidence interval of the estimate of $E$. This confirms that the very high values of $E_{a w}$ have little influence on estimated total respiratory elastance.

Using eqn. 8 we estimated an $E_{c w}$ of $10.00 \mathrm{cmH}_{2} \mathrm{Ol}^{-1}$ and then we calculated an $E_{p}$ of $11.13 \mathrm{cmH}_{2} \mathrm{O} \mathrm{l}^{-1}$, as the difference between the estimates of $E$ and $E_{c w}$. 
Also in this case (table 1) we see that the estimates of $E_{c w}$ is exactly equal to the true value of the forward model, as expected, while the estimate of $E_{p}$ reasonably approximates the model simulated value.

Table 1: Parameter value set for the forward model of breathing mechanics.

\begin{tabular}{|l|c|}
\hline$K_{1}\left(\mathrm{cmH}_{2} \mathrm{O} \mathrm{s} \mathrm{l}^{-1}\right)$ & 0.5 \\
\hline$K_{2}\left(\mathrm{cmH}_{2} \mathrm{O} \mathrm{s}^{2} ~^{-2}\right)$ & 0.2 \\
\hline$K_{3}\left(\mathrm{cmH}_{2} \mathrm{O} \mathrm{s} \mathrm{l}^{-1}\right)$ & 0.2 \\
\hline$v_{c \max }(\mathrm{l})$ & 0.1 \\
\hline$K_{4}\left(\mathrm{cmH}_{2} \mathrm{O} \mathrm{s}^{-1}\right)$ & 47.5 \\
\hline$a\left(\mathrm{cmH}_{2} \mathrm{O}^{-1}\right)$ & 1.05 \\
\hline$b\left(\mathrm{cmH}_{2} \mathrm{O}\right)$ & 6.0 \\
\hline$F R C(\mathrm{l})$ & 3.0 \\
\hline$E_{p}\left(\mathrm{cmH}_{2} \mathrm{O} \mathrm{l}^{-1}\right)$ & 10.0 \\
\hline$E_{c w}\left(\mathrm{cmH}_{2} \mathrm{O} \mathrm{l}^{-1}\right)$ & 10.0 \\
\hline
\end{tabular}

Table 2: Model parameter estimates and corresponding 95\% confidence interval during inspiration.

\begin{tabular}{|c|c|c|}
\hline$R_{\text {insp }}\left(\mathrm{cmH}_{2} \mathrm{O} \mathrm{s} \mathrm{l}^{-1}\right)$ & 13.55 & $12.79,14.31$ \\
\hline$E\left(\mathrm{cmH}_{2} \mathrm{O} \mathrm{l}^{-1}\right)$ & 21.13 & $19.59,22.66$ \\
\hline
\end{tabular}

Finally, using mouth flow data during EFL detected by NEP, we determined the unknown parameter $A$ of eqn. 14 and, from the estimated values of $A$ and $E_{p}$, the value of $R_{d}$. The estimate of $R_{d}$ was equal to $15.54 \mathrm{cmH}_{2} \mathrm{O} \mathrm{s} \mathrm{l}^{-1}$, with a $95 \%$ confidence interval of $15.36-15.73 \mathrm{cmH}_{2} \mathrm{O} \mathrm{s} \mathrm{l}^{-1}$.

If we are to interpret the estimate of $R_{d}$ on the basis of the forward model of fig. 2, it is convenient to observe the time course of $R_{l}$ over a whole breathing cycle (fig. 4). As expected, $R_{l}$ decreases during inspiration $(0-0.9 \mathrm{~s})$, remains about constant during the post-inspiratory pause (0.9-1 s) and increases during expiration (1-4 s). The continuous line shows the part of expiration characterized by EFL and in this time interval, $R_{l}$ increases from about 12.5 to $14 \mathrm{cmH}_{2} \mathrm{O} \mathrm{s} \mathrm{l}^{-1}$. The corresponding mean is $13.43 \mathrm{cmH}_{2} \mathrm{O} \mathrm{s} \mathrm{l}^{-1}$.

The estimate of $R_{d}$ is about $15 \%$ greater than the mean value of $R_{l}$ during EFL, indicating that the pressure at the left end of $R_{l}$ (fig. 2 ) is greater than $p_{p l}$. This finding is confirmed by the time course of the transmural pressure across $E_{a w}$ (fig. 5), showing that $p_{t m}$ does not reach zero at any time during the flowlimited expiration. In this simulated case, EPP is therefore located downstream 
of $R_{l}$ and the proposed estimation approach rightly estimates a value for $R_{d}$ greater than the forward model lower resistance $R_{l}$.

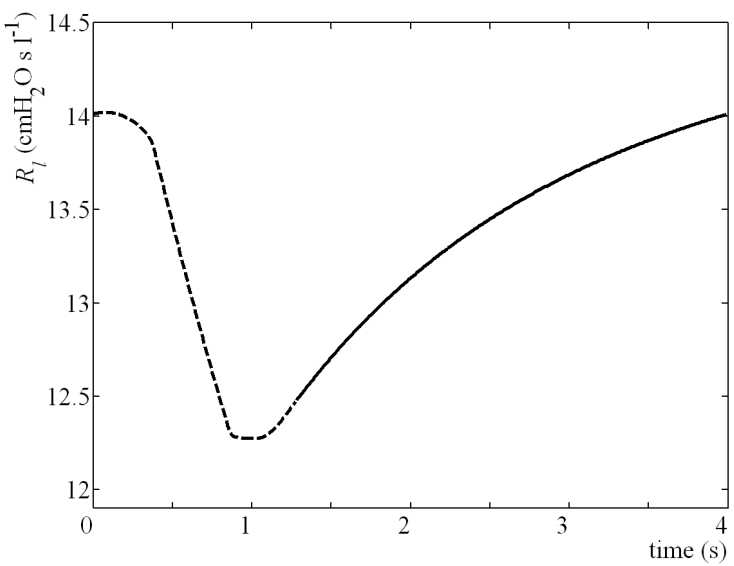

Figure 4: $\quad$ Model simulated time course of lower resistance $\left(R_{l}\right)$.

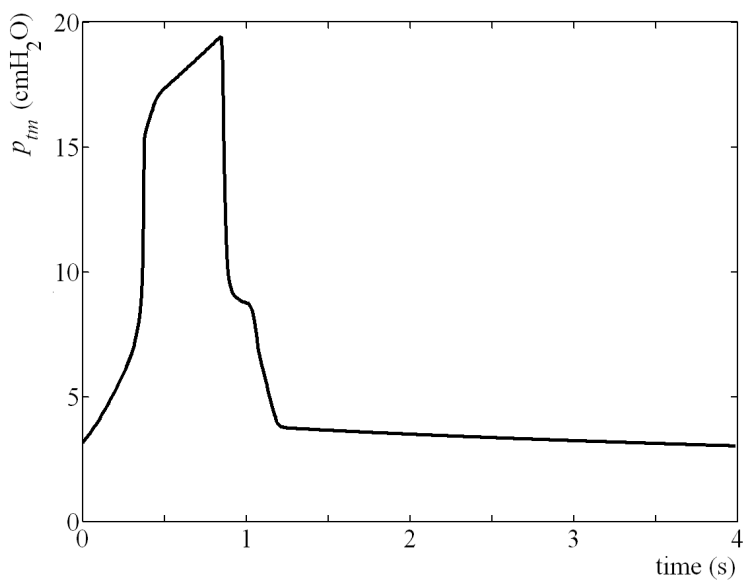

Figure 5: $\quad$ Model simulated time course of transmural pressure $\left(p_{t m}\right)$ across $E_{a w}$.

In conclusion, the proposed approach seems to provide a reliable estimate of deep resistances that determine flow during expiration in mechanically ventilated flow-limited patients. Though model-to-model analysis is useful to assess the performance of the method, the results need further confirmation with clinical experimental data. 


\section{Acknowledgment}

This work was supported by the Italian Ministry of Education, University and Research (MIUR).

\section{References}

[1] West, J.B., Pulmonary Pathophysiology-The Essentials, Williams \& Wilkins: Baltimore, 1998.

[2] Valta, P., Corbeil, C., Lavoie, A., Campodonico, R., Koulouris, N., Chasse, M., Braidy, J. \& Milic-Emili, J., Detection of expiratory flow limitation during mechanical ventilation. Am. J. Respir. Crit. Care Med., 150(5Pt1), pp. 1311-1317, 1994.

[3] Barbini, P., Brighenti, C., Cevenini, G. \& Gnudi, G., A dynamic morphometric model of the normal lung for studying expiratory flow limitation in mechanical ventilation. Ann. Biomed. Eng., 33(4), pp. 516528, 2005.

[4] Hyatt, R.E., Rodarte, J.R., Wilson, T.A. \& Lambert, R.K., Mechanisms of expiratory flow limitation. Ann. Biomed. Eng., 9(5-6), pp. 489-499, 1981.

[5] Barbini, P., Cevenini, G. \& Avanzolini, G, Nonlinear mechanisms determining expiratory flow limitation in mechanical ventilation: a modelbased interpretation. Ann. Biomed. Eng., 31(8), pp. 908-916, 2003.

[6] Officer, T.M., Pellegrino, R., Brusasco, V. \& Rodarte, J.F., Measurement of pulmonary resistance and dynamic compliance with airway obstruction. J. Appl. Physiol., 85(5), pp. 1982-1988, 1998.

[7] Barbini, P., Cevenini, G, Bernardi, F., Massai, M.R, Gnudi, G. \& and Avanzolini, G., Effect of compliant intermediate airways on total respiratory resistance and elastance in mechanical ventilation. Med. Eng. Phys., 23(3), pp. 185-194, 2001.

[8] Kaczka, D.W, Ingenito, E.P., Body, S.C., Duffy, S.E., Mentzer, S.J., DeCamp, M.M. \& Lutchen, K.R, Inspiratory lung impedance in COPD: effects of PEEP and immediate impact of lung volume reduction surgery. J. Appl. Physiol., 90 (5), pp. 1833-1841, 2001.

[9] Kaczka, D.W, Ingenito, E.P. \& Lutchen, K.R, Technique to determine inspiratory impedance during mechanical ventilation: implications for flow limited patients. Ann. Biomed. Eng., 27(3), pp. 340-355, 1999.

[10] Pepe, P.E. \& Marini, J.J., Occult positive end-expiratory pressure in mechanically ventilated patients with airflow obstruction: the auto-PEEP effect. Am. Rev. Respir. Dis., 126(1), pp.166-170, 1982. 